\title{
To study the clinical profile, management and outcome of diabetic pregnancies in a rural tertiary care institute of Punjab
}

\author{
Reena Sood*, Parvinder Arora, Madhu Nagpal
}

Department of Obstetrics and Gynecology, Sri Guru Ram Das Institute of Medical Sciences and Research, Amritsar, Punjab, India

Received: 21 August 2017

Accepted: 24 August 2017

*Correspondence:

Dr. Reena Sood,

E-mail: dr.reenasood@gmail.com

Copyright: (C) the author(s), publisher and licensee Medip Academy. This is an open-access article distributed under the terms of the Creative Commons Attribution Non-Commercial License, which permits unrestricted non-commercial use, distribution, and reproduction in any medium, provided the original work is properly cited.

\section{ABSTRACT}

Background: Diabetes mellitus is a disorder of carbohydrate metabolism. In India, about 50.9 million people suffer from diabetes, and this figure is likely to go up to 80 million by 2025 , making it the 'diabetes capital' of the world. GDM develops due to an inability to compensate for physiological increase in insulin resistance that develops progressively throughout pregnancy as a consequence of multiple factors including placental hormones, increased caloric intake and reduced physical activity. Many studies report increased incidence of adverse foetal and maternal outcome in diabetic pregnancy.

Methods: The present study is a retrospective cum prospective analysis carried out in SGRDIMSAR from 1st May 2015 to 30th April 2017. Patients with diabetic pregnancies who had delivery in our institution were included.

Results: Total number of diabetic pregnancies during the study period were 76 . Out of 76 patients 15 were PGDM and 61 were GDM. Maximum no of patients in PG group were in age group of 31-35 years, while in GDM group were upto 30 years of age. Gestational age at diagnosis in PGDM group is $13.42 \pm 1.2$ weeks versus $29.62 \pm 4.53$ weeks. Maximum patients $(70.5 \%)$ in GDM group were managed by diet and exercise, while in PGDM group maximum patients $(93.3 \%)$ needed insulin for glycemic control. Maternal complications were maximum in PGDM group. Most common complication was hypertensive disorders of pregnancy. Mean gestational age at delivery was $35.15 \pm 1.42$ weeks and 37.06 \pm 1.29 weeks in PGDM and GDM respectively ( $\mathrm{p}$ value $=0.00$ ). $11.8 \%$ neonates had a birth weight of $>4 \mathrm{~kg}$ (macrosomia). 77\% of neonates in GDM group had an uneventful outcome.

Conclusions: Considering rising incidence and magnitude of the problem and its complications, identification and treatment of diabetic pregnancy is the need of hour.

Keywords: Diabetes mellitus, Outcome, Pregnancy, Treatment

\section{INTRODUCTION}

Diabetes Mellitus is a disorder of carbohydrate metabolism. In 2008, an estimated 347 million people in the world had diabetes and the prevalence is growing, particularly in low- and middle-income countries. Diabetes was chosen as the theme for World Health Day in 2016. ${ }^{1}$ In India, about 50.9 million people suffer from diabetes, and this figure is likely to go up to 80 million by
2025, making it the 'diabetes capital' of the world. ${ }^{2}$ The increasing prevalence in developing countries is related to increasing urbanization, decreasing levels of physical activity, changes in dietary patterns and increasing prevalence of obesity. ${ }^{3}$ Traditional definition of Gestational diabetes mellitus (GDM), which is still used by American College of Obstetrics and Gynecology, is any degree of glucose intolerance that either commences or is first diagnosed during pregnancy. This definition 
applies regardless of whether treatment involves insulin or diet modification alone. In 2010, the International Association of Diabetes and Pregnancy Study Group (IADPSG) recommended a new terminology that diabetes that is first recognised in pregnancy can be classified as either overt or gestational. This recognises that many women have unrecognised type $2 \mathrm{DM}$ at the time of conception. GDM develops due to an inability to compensate for physiological increase in insulin resistance that develops progressively throughout pregnancy as a consequence of multiple factors including placental hormones, increased caloric intake and reduced physical activity. Prevalence of GDM directly reflects that of type 2 diabetes in given population. ${ }^{4}$ There are a lot of conflicting guidelines and protocols for its screening, diagnosis and treatment. The reasons cited for rising incidence are change in lifestyle and dietary habits, older age at first conception, polycystic ovarian disease, obesity and more so increased awareness and changing methodology in testing for the condition. ${ }^{5}$ Many studies report increased incidence of adverse foetal and maternal outcome in diabetic pregnancy. So, screening and managing women at appropriate gestational age is important to minimize these adverse outcomes. Glycaemic control can safely be achieved with a combination of exercise, nutritional and pharmaceutical interventions. Aim of present study is to evaluate clinical profile of diabetic pregnancies, interventions needed and maternal and neonatal outcome.

\section{METHODS}

The present study is a retrospective cum prospective analysis carried out in SGRDIMSAR from 1st May 2015 to 30th April 2017. Patients with diabetic pregnancies who had delivery in our institution were included. Patients having some endocrinopathy as thyroid disorder were excluded from the study. Our Institution is a tertiary care centre situated in rural area in the periphery of Amritsar, Punjab. Routinely as a protocol in all booked pregnancies we are doing fasting blood sugar levels (FBS) or random blood sugar (RBS) at booking visit and then screening OGTT (Oral Glucose Tolerance Test) at 26-28 weeks of gestation as per IADPSG (International Association of Diabetes and Pregnancy Study group Guidelines). ${ }^{6}$ In certain high risk groups (as history of macrosomia in previous pregnancies, previous unexplained still birth, polycystic ovarian syndrome, obesity) the OGTT test is done at earlier gestational age. This test is done after 8 hours of fasting. First sample taken is of fasting blood sugar followed by intake of 75 gm glucose solution orally and blood sugar levels estimated again at 1 hour and 2 hours.

Patients are grouped according to Whites classification into gestational diabetes mellitus (GDM) and pregestational diabetes mellitus (PGDM). Patients givng history of DM at first visit or found to be having FBS > $126 \mathrm{mg} / \mathrm{dl}$ or $\mathrm{RBS}>200 \mathrm{mg} / \mathrm{dl}$ in first trimester were included in PGDM group.
Each case record was analysed in detail with special emphasis on maternal profile, clinical presentation, modality and gestational age of diagnosis, HbA1c values, treatment given, maternal complications if any and neonatal status.

Blood sugar cut off values are given in table.

Table 1: IADPSG Criteria for a positive $75 \mathrm{gm}$ OGTT in pregnancy (24-28 weeks gestation).

\begin{tabular}{|c|c|c|c|}
\hline & $\begin{array}{l}\text { Fasting } \\
\text { plasma } \\
\text { glucose }\end{array}$ & $\begin{array}{l}1 \text { hour } \\
\text { plasma } \\
\text { glucose }\end{array}$ & $\begin{array}{l}2 \text { hours } \\
\text { plasma } \\
\text { glucose }\end{array}$ \\
\hline $\begin{array}{l}\text { IADPSG } \\
\text { and } \\
\text { American } \\
\text { Diabetes } \\
\text { Association }\end{array}$ & $\geq 92 \mathrm{mg} / \mathrm{dl}$ & $\begin{array}{l}\geq 180 \\
\mathrm{mg} / \mathrm{dl}\end{array}$ & $\geq 153 \mathrm{mg} / \mathrm{dl}$ \\
\hline
\end{tabular}

All diabetic pregnancies have been managed by a multidisciplinary team involving obstetrician, physician, dietician, ophthalmologist and paediatrician. After taking approval from institution ethics committee, data was retrieved by reviewing obstetric admission register, birth register and case records. Data was compiled and statistically analysed using SPSS version 23 applying Chi square and t tests.

\section{RESULTS}

Total number of diabetic pregnancies during the study period were 76 . Out of 76 patients 15 were PGDM and 61 were GDM.

Table 2: Maternal age distribution in both groups.

\begin{tabular}{|lllll|}
\hline Maternal age & PG DM & & GDM & \\
\hline & N (15) & \% & N (61) & \% \\
\hline $20-25$ & 0 & 0.00 & 24 & 39.34 \\
\hline $26-30$ & 5 & 33.33 & 24 & 39.34 \\
\hline $31-35$ & 7 & 46.67 & 11 & 18.03 \\
\hline$>35$ & 3 & 20.00 & 2 & 3.28 \\
\hline
\end{tabular}

Maximum no of patients in PG group were in age group of 31-35 years, while in GDM group were upto 30 years of age. Mean age in PG group was $32.20 \pm 4.3$ years while mean age in GDM group was $27.86 \pm 5.0$ years. After applying $\mathrm{t}$ - test $\mathrm{p}$ value $=0.00$ which is significant .

Table 3: distribution of parity in both groups.

\begin{tabular}{|lllll|}
\hline & PG & \multicolumn{3}{c|}{ GDM } \\
\hline & No & $\%$ & No & $\%$ \\
\hline Primigravidas & 2 & 13.33 & 20 & 32.79 \\
\hline Multigravias & 13 & $86.7 \%$ & 41 & $67.2 \%$ \\
\hline
\end{tabular}


Among PGDM 2 (13.3\%)were primigravidas and 13 $(86.7 \%)$ were multigravidas. Among GDM group 20 $(32.8 \%)$ were primigravidas and 41 (67.2\%) were multigravidas ( $\mathrm{p}$ value $=0.137$ insignificant $)$.

In PGDM group $13.3 \%$ patients had history of PCOD and previous IUFD.

Table 4: Prior obstetric performance in both groups.

\begin{tabular}{|lll|ll|}
\hline H/O PCOS & 2 & 13.33 & 2 & 3.28 \\
\hline Prev IUD & 2 & 13.33 & 3 & 4.9 \\
\hline Prev stillborn & 1 & 6.67 & 1 & 1.64 \\
\hline Prev abortion & 1 & 6.67 & 0 & 0.00 \\
\hline $\begin{array}{l}\text { Prev h/o } \\
\text { macrosomia }\end{array}$ & 1 & 6.67 & 1 & 1.64 \\
\hline
\end{tabular}

Gestational age at diagnosis in PGDM group is $13.42 \pm 1.2$ weeks versus $29.62 \pm 4.53$ weeks. on applying $t$ - test $\mathrm{p}$ value is 0.02 (significant).

HbA1c at diagnosis was $<6.5$ in $77.05 \%$ of patients in GDM group and in only $13.33 \%$ in PGDM.

Table 5: HbA1c in both groups.

\begin{tabular}{|l|llll|}
\hline & PG & \multicolumn{3}{c|}{ GDM } \\
\hline & No. & $\%$ & No. & \% \\
\hline$\leq 6.5$ & 2 & 13.33 & 47 & 77.05 \\
\hline$>6.5$ & 13 & 86.67 & 14 & 22.95 \\
\hline
\end{tabular}

After diagnosis, patients were evaluated for renal function, fundus changes. Dietician and physician opinion was taken and patients were advised diet and exercise. After 2 weeks of dietary management, if glycemic targets (FBS <95 mg/dl and 2-hour PPBS < $120 \mathrm{mg} / \mathrm{dl}$ ) were not met, inpatient treatment was done in the initial stages which involved regular blood sugar levels monitoring and training of the patients for selfblood glucose monitoring and insulin injections if required. At the time of discharge, they were asked to keep a written record of blood sugar levels at home and bring it at subsequent OPD visits. They were explained the importance of appropriate diet and daily walks in ensuring the better neonatal outcome.

Patients with initial high levels were monitored by HbA1c levels 3 monthly till delivery. Glucocorticoids were given for lung maturity in cases who developed some complications as hypertensive disorder of pregnancy. The injections were given as inpatient only and glycemic control was ensured with higher dosage of insulin. Patients who were controlled with diet and exercise pregnancy was continued till 40 weeks. Those on pharmacotherapy were routinely advised admission at 36 weeks for safe confinement. Those with some comorbidities as hypertension needed earlier admission and supervision.
Maximum patients $(70.5 \%)$ in GDM group were managed by diet and exercise, while in PGDM group maximum patients $(93.3 \%)$ needed insulin for glycemic control. Only $24.6 \%$ of patients needed insulin for management in GDM group. $6.7 \%$ and $1.64 \%$ patients in PGDM and GDM group respectively received a combination of OHA and insulin. All these patients had history of PCO and prior intake of metformin. 2 patients in GDM group who had started metformin prior to pregnancy had good control with that only. None of the patients was given any other OHA apart from metformin in our study.

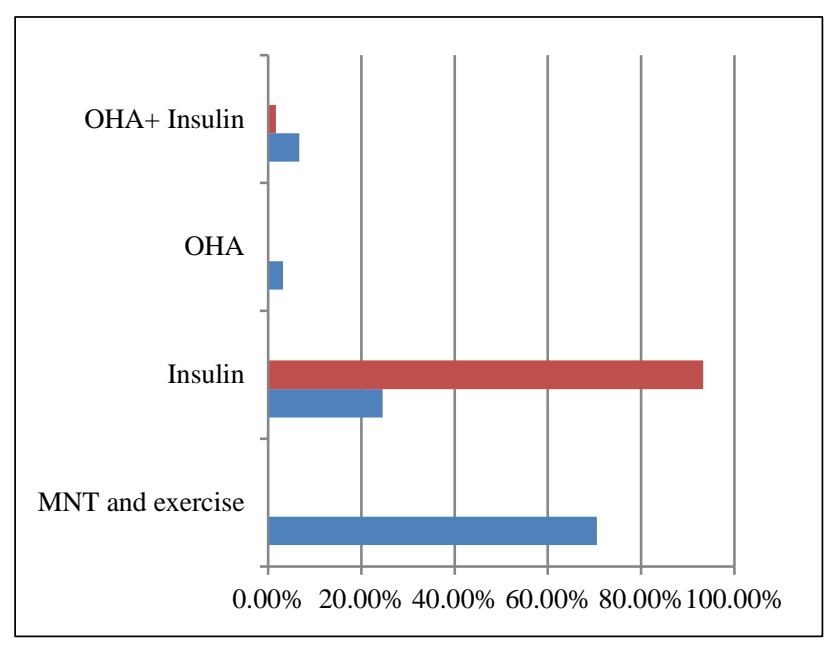

Figure 1: Treatment modalities in both groups. GDM (blue) PG (pink).

Maternal complications were maximum in PGDM group. Most common complication was hypertensive disorders of pregnancy. 2 patients in PGDM group had developed pancreatitis in pregnancy. Both these patients were obese and had uncontrolled diabetes. One of these patients had IUFD also.

Table 6: Maternal complications in the pregnancy.

\begin{tabular}{|lllll|}
\hline \multicolumn{1}{|c}{ PGDM } & GDM \\
\hline & No. & $\%$ & No. & $\%$ \\
\hline PIH & 3 & 20.00 & 1 & 1.64 \\
\hline Pre-eclampsia & 4 & 26.67 & & 0.00 \\
\hline Eclampsia & 0 & 0.00 & 1 & 1.64 \\
\hline Pancreatitis & 2 & 13.33 & & 0.00 \\
\hline Polyhydramnios & 3 & 20.00 & 3 & 4.92 \\
\hline $\begin{array}{l}\text { No maternal } \\
\text { complication }\end{array}$ & 3 & 20 & 56 & 91.8 \\
\hline
\end{tabular}

Most common mode of deliveries in both groups was LSCS. In PG group $26.7 \%$ patients had vaginal delivery, in GDM group $32.8 \%$ patients had vaginal delivery.

In 4 cases, out of $11(36.4 \%)$ indication of caesarean section in PGDM was previous caesarean, in another 4 cases $(36.4 \%)$ it was macrosomia, in 2 cases $(18 \%)$ was severe preeclampsia and in $0.45 \%$ was malpresentation. 
Table 7: Mode of delivery in both groups.

\begin{tabular}{|lllll|}
\hline & PGDM & GDM \\
\hline NVD & No. & $\%$ & No. & $\%$ \\
\hline Instrumental & 3 & 20 & 17 & 27.87 \\
\hline LSCS & 1 & 6.67 & 3 & 4.92 \\
\hline
\end{tabular}

In GDM group in 12 out of 41cesareans (29.2\%) indication was previous Caesarean section, 10 cases (24\%) was cephalopelvic disproportion with failure of progress of labour, 10 cases $(24 \%)$ had fetal distress indicated as non-assuring cardiotocography, in 5 out $12.2 \%$ it was macrosomia,

2 cases $(4.8 \%)$ had placenta previa and in another 2 $(4.8 \%)$ it was malpresentation.

Mean gestational age at delivery was $35.15 \pm 1.42$ weeks and $37.06 \pm 1.29$ weeks in PGDM and GDM respectively $(\mathrm{p}$ value $=0.00)$.

Table 8: Gestational age of delivery in weeks.

\begin{tabular}{|lllll|}
\hline & PGDM & GDM \\
\hline & No. & $\%$ & No. & $\%$ \\
\hline $\begin{array}{l}\text { Moderate preterm } \\
\text { (32-33.6 week) }\end{array}$ & 2 & 13.3 & & \\
\hline $\begin{array}{l}\text { Late preterm (34- } \\
\text { 36.6 week) }\end{array}$ & 9 & 60.0 & 24 & 39.3 \\
\hline Term > 37 weeks & 4 & 26.7 & 37 & 60.7 \\
\hline
\end{tabular}

Higher incidence of preterm births in PGDM was mainly due to associated hypertensive disorders and polyhydramnios.

Though $80.0 \%$ neonates in PGDM and $57.38 \%$ neonates in GDM group needed nursery observation, this was primarily for the blood sugar monitoring. $77 \%$ of neonates in GDM group had an uneventful outcome.

Table 9: Neonatal complications in both groups.

\begin{tabular}{|lllll|}
\hline & PGDM & \multicolumn{3}{l|}{ GDM } \\
\hline $\begin{array}{l}\text { Nongenital } \\
\text { malformations }\end{array}$ & 1 & 6.67 & 1 & 1.64 \\
\hline IUFD & 1 & 6.67 & 3 & 4.92 \\
\hline $\begin{array}{l}\text { Neonatal } \\
\text { hypoglycemia }\end{array}$ & 1 & 6.67 & 1 & 1.64 \\
\hline Respiratory distress & 6 & 40.00 & 9 & 14.75 \\
\hline Uneventful & 6 & 40.0 & 47 & 77.0 \\
\hline
\end{tabular}

One IUFD in PGDM group was in an uncontrolled diabetic, obese patient who had developed pancreatitis also. 2 patients in GDM group who had IUFD were unbooked cases diagnosed after admission. Unfortunately, one booked patient diagnosed with GDM on insulin treatment had IUFD at 36 weeks.
In our study 9 out $76(11.8 \%)$ neonates had a birth weight of $>4 \mathrm{~kg}$ (macrosomia). ${ }^{7}$ In PGDM 4 cases $(26.7 \%)$ had macrosomia. In GDM group 5 neonates $(8.2 \%)$ had macrosomia. However, on plotting the birth weights against gestational age at delivery on a graph according to Fentons growth chart, $11(73.33 \%)$ neonates in PGDM group and $28(45.90 \%)$ neonates in GDM group were found to be weighing $>90$ th percentile $(\mathrm{LGA}){ }^{8}$

\section{DISCUSSION}

As early as in the 1940s, it was recognized that women who developed diabetes years after pregnancy had experienced abnormally high fetal and neonatal mortality. By the 1950s the term "gestational diabetes" was applied to what was thought to be a transient condition that affected fetal outcomes adversely, then abated after delivery. ${ }^{9}$ In 1989, a group of patient representatives, governmental representatives and diabetes experts met in St. Vincent, Italy, to discuss the growing problem of diabetes across Europe. The meeting, which was organised under the auspices of the World Health Organisation and the International Diabetes Federation resulted in the St. Vincent Declaration which aimed at Achieving pregnancy outcome in the diabetic woman that approximates that of the non-diabetic woman. ${ }^{10}$ The prevalence of GDM in India has been reported ranging from $9.8 \%-18 \% .^{11,12}$

In our study mean age in PGDM group was 32.20 44.36 years while mean age in GDM group was $26.86 \pm 5$ years ( $\mathrm{p}$ value $=0.00$ ). So mean age is higher in PGDM group as compared to GDM. This result is consistent with the result of Pandey $U$ et al but not with the study by Shefali et al. ${ }^{12,13}$ Higher incidence of recurrent abortions has been found in earlier studies but in our study previous abortions were found only in $6.67 \%$ in PGDM group. ${ }^{12,13}$

Mean GA at Diagnosis was $13.42 \pm 1.2$ weeks in PGDM group and 29.62 \pm 4.53 in GDM group found to be in coherence with previous studies. ${ }^{12}$

Most common maternal complication observed in the study was hypertensive disorder of pregnancy which is again consistent with earlier study. ${ }^{15,16}$ Two patients in PGDM group had pancreatitis that can be because of associated maternal obesity and hyperlipidemia.

$91.8 \%$ of patients in GDM group did not have any maternal complications. Maximum patients $(70.5 \%)$ in GDM group were managed by diet and exercise while maximum patients in PGDM (93.3\%) required insulin for glycemic control. This is similar to previous study results. $^{13}$

OHA (Metformin) was used only in those patients who were taking it prior to pregnancy. Though safety of metformin has been proved by earlier authors. ${ }^{17}$ In our institute we are still using Insulin as a therapeutic measure when glycemic targets are not achieved by diet 
and exercise. We are not using any other OHA than metformin in pregnancy. However, in a small number of patients taking metformin no maternal or foetal untoward effects were seen.

In both groups incidence of caesarean section was high (73.3\% in PGDM and $67.21 \%$ in GDM) this is similar to earlier studies. ${ }^{13,15}$

Mean gestational age at delivery was lower in PGDM as compared to GDM in our study ( $p$ value $=0.00$ ) this can be due to higher incidence of associated comorbidities in PGDM group which is consistent with results of previous studies. ${ }^{13}$ Positive trend towards prematurity in neonates of diabetic mothers has been found in previous studies. ${ }^{16}$

Incidence of macrosomia (Birth weight $>4 \mathrm{~kg}$ ) in our study is $11.8 \%$. But $51.3 \%$ neonates in our study were LGA according to Fenton chart. $^{8}$ These results are consistent with earlier studies. ${ }^{13,14,16}$

As proven by earlier studies women with PGDM are at greater risk of unfavorable pregnancy outcomes than GDM. Pregnancy outcomes also depend on glycemic control. ${ }^{14}$

The use of the IADPSG criteria results in increase in the rate of GDM, but this also appears to identify additional women at risk of adverse pregnancy outcome. 12,18 Results of Landon et al study (2009) has concluded that the treatment of mild gestational diabetes reduces the risk of fetal overgrowth, shoulder dystocia, caesarean delivery and hypertensive disorders.19 Also the long-term outcome of children born to diabetic mothers has shown a higher incidence of metabolic syndrome. 20 Preconceptional counselling should be done in PGDM so that they enter pregnancy in an optimal stage. Dietary modifications and exercises should be promoted in the risk groups of GDM. Studies have concluded that higher level of physical activity before pregnancy or early in pregnancy are associated with significantly lower risk of developing GDM. ${ }^{21}$

Considering rising incidence and magnitude of the problem and its complications, identification and treatment of diabetic pregnancy is the need of hour. As we are living in an era of sedentary lifestyle and its sequelae, rather than detection and treatment the focus should be shifted to preventive measures like dietary modifications and physical exercise in pre pregnancy and early pregnancy period. Preconceptional counselling should be encouraged in already diagnosed diabetics, PCOS and obese patients to have a better outcome.

Limitations of this study was single center retrospective study.

Funding: No funding sources Conflict of interest: None declared
Ethical approval: The study was approved by the Institutional Ethics Committee

\section{REFERENCES}

1. World Health Organization, World Health Day Diabetes, 2016. Available at www.searo.who.int/india/mediacentre/events/2016/e $\mathrm{n} /$.

2. Diabetes Foundation India - DFI. Available at www.diabetesfoundationindia.org/about.htm.

3. Rajput M, Bairwa M, Rajput R. Prevalence of gestational diabetes mellitus in rural Haryana: A community-based study. Indian $\mathrm{J}$ Endocrinol Metabol. 2014;18(3):350.

4. Studd J, Tan SL, Chervenak FA. India:Tree Life Media. Current Progress in Obstetr Gynaecol. 2012;1:37.

5. Bhide A, Arulkumaran S, Damania K, Daftary S. Arias' Practical Guide to High Risk Pregnancy and Delivery - A South Asian Perspective. Elsevier; 2016:254.

6. International Association of Diabetes and Pregnancy Study Groups Consensus Panel. International association of diabetes and pregnancy study groups recommendations of the diagnosis and classification of hyperglycaemia in pregnancy. Diabetes Care. 2010;33:676-82.

7. Kc K, Shakya S, Zhang H. Gestational diabetes mellitus and macrosomia: a literature review. Ann Nutr Metab. 2015;66 Suppl 2:14-20.

8. Fenton T, Kim J. A systematic review and metaanalysis to revise the Fenton growth chart for preterm infants. BMC Pediatr. 2013;13:59.

9. Buchanan T, Xiang A. Gestational diabetes mellitus. J Clin Investigat. 2005;115(3):485-91.

10. Vincent declaration on the treatment of diabetes Management Available at https://www.diapedia.org > Management , Monitoring and organisation of diabetes care.

11. Seshiah V, Balaji V, Balaji MS. Gestational diabetes mellitus in India. $\mathrm{J}$ Assoc Physicians India. 2004;52:707-11.

12. Bhavadharini B, Mahalakshmi MM, Anjana RM, Maheswari K, Uma R, Deepa M, et al. Prevalence of gestational diabetes mellitus in urban and rural Tamil Nadu using IADPSG and WHO 1999 criteria (WINGS 6). Clin Diabetes Endocrinol. 2016;5(2):8.

13. Pandey U, Agrawal N, Agrawal S, Batra S. Outcome of diabetic pregnancies in a tertiary referral centre, Varanasi. J Obstet Gynecol India. 2015;66(4):22632.

14. Shefali AK, Kavitha M, Deepa R. Preganacy outcomes in pregestational and gesatational diabetic women in comparison to non- diabetic women. Aprospectivetudy in Asian Indian mothers. J Assoc Physicians India. 2006;54:613-8.

15. Saxena P, Tyagi S, Prakash A. Pregnancy outcome of women with gestational diabetes in a tertiary level 
hospital of North India. Indian J Community Med. 2011;36(2):120-3.

16. Bhat M, Ramesha K, Sarma S, Menon S, Ganesh Kumar S. Outcome of gestational diabetes mellitus from a tertiary referral center in South India: a casecontrol study. J Obstet Gynecol India. 2012;62(6):644-649.

17. Magon N, Seshiah V. Gestational diabetes mellitus: Non-insulin management. Indian $\mathrm{J}$ Endocrinol Metabol. 2011;15(4):284.

18. Mayo K, Melamed N, Vandenberghe H, Berger H. The impact of adoption of the International Association of Diabetes in Pregnancy Study Group criteria for the screening and diagnosis of gestational diabetes. Am J Obstetr Gynecol. 2015;212(2):224.e1-224.e9.

19. Landon M. A multicenter, randomized trial of treatment for mild gestational diabetes. $\mathrm{N}$ Engl J Med. 2009;361:1339-48.
20. Boney C. Metabolic syndrome in childhood: association with birth weight, maternal obesity, and gestational diabetes mellitus. Pediatr. 2005;115(3):e290-296.

21. Tobias D, Zhang C, van Dam RM, Bowers $\mathrm{K}, \mathrm{Hu}$ FB. Physical activity before and during pregnancy and risk of gestational diabetes mellitus. Diabetes Care. 2011;34(1):223-9.

Cite this article as: Sood R, Arora P, Nagpal M. To study the clinical profile, management and outcome of diabetic pregnancies in a rural tertiary care institute of Punjab. Int J Reprod Contracept Obstet Gynecol 2017;6:4323-8. 